\title{
EPQ Policies Considering the Holding Cost of Raw Materials under Conditions of Two-Level Trade Credit and Limited Storage Capacity
}

\author{
Ghi-Feng Yen'1, Shy-Der Lin², An-Kuo Lee ${ }^{1}$ \\ ${ }^{1}$ Department of Business Administration, Chung Yuan Christian University, Taiwan \\ ${ }^{2}$ Department of Applied Mathematics, Chung Yuan Christian University, Taiwan \\ Email: gfyen@cycu.edu.tw, shyder@cycu.edu.tw,dreamair@icloud.com
}

How to cite this paper: Yen, G.-F., Lin, S.-D. and Lee, A.-K. (2019) EPQ Policies Considering the Holding Cost of Raw Materials under Conditions of Two-Level Trade Credit and Limited Storage Capacity. Open Access Library Journal, 6: e5140. https://doi.org/10.4236/oalib.1105140

Received: December 24, 2018

Accepted: January 27, 2019

Published: January 30, 2019

Copyright $\odot 2019$ by author(s) and Open Access Library Inc.

This work is licensed under the Creative Commons Attribution International License (CC BY 4.0).

http://creativecommons.org/licenses/by/4.0/

\begin{abstract}
The economic production quantity model is widely used as a decision making tool. However, climate change and energy supply instability have raised the transport costs of raw materials, thereby affecting production costs. Therefore, this paper presents a new model that considers the holding costs of raw materials under conditions of two-level trade credit and limited storage capacity. Four theorems are developed to characterize the optimal solutions according to a cost-minimization strategy. Finally, a sensitivity analysis is executed to investigate the effects of the various parameters on ordering policies and the annual total relevant costs.
\end{abstract}

\section{Subject Areas}

Applied Statistical Mathematics, Business Analysis

\section{Keywords}

Economic Production Quantity (EPQ), Raw Materials, Trade Credit, Limited Storage Capacity

\section{Introduction}

The economic order quantity (EOQ) model [1] and economic production quantity (EPQ) model [2] have been widely used in mathematical analysis and inventory management. Scholars have focused on the holding costs in production and sale process, but have ignored the holding costs of raw materials. However, the supplier provision of raw materials is affected by many factors such as the sale date and weather, thus affecting the price fluctuation of raw materials. 
Therefore, [3] modified the EPQ model to consider the holding costs of raw materials, and [4] [5] subsequently incorporated raw materials into EPQ model.

[6] established an EOQ model under the condition of permissible delay. [7] proposed the following trade credit condition: If a customer buys one item from a retailer at time $t \in[0, T]$, then the customer will have a trade credit period $N-t$ and make the payment at time $N$. Therefore, the retailer allows a maximal trade credit period $N$ for the customer to settle the account [8] [9] [10] [11] [12]. The retailer purchases more quantities that exceed what the owned warehouse (OW) would consider for storing in a rented warehouse (RW) [9] [13] [14] [15] [16].

[9] developed an EPQ model that considers two-level of trade credit-the finite replenishment rate and limited storage capacity-but it ignores the effects of raw materials and annual purchasing costs. Therefore, the main purpose of this paper is to include the holding costs of raw materials for determining optimal inventory policies, two-level trade credit and limited storage. This paper considers a structure that involves three stages, each from the "supplier, manufacturer, and customer (retailer)". This paper assumes the manufacturer's position to calculate the annual total relevant costs, including the ordering cost, purchasing costs, holding costs, interest payable, and interest earned. According to the cost-minimization strategy, four theorems are developed to characterize the optimal solution. Finally, sensitivity analysis is performed to determine the critical impact factors and draw the conclusions. For business managers, the model is simple to use for calculating the optimal cycle and annual total relevant costs. Accurate information is crucial to making such decisions as a basis for an investment plan.

\section{Notations and Assumptions}

\subsection{Notations}

$Q$ order size;

$P$ production rate;

$D$ demand rate;

$A$ ordering cost;

$T$ cycle time.

$\rho=1-\frac{D}{P}>0$

$L_{\max }$ storage maximum;

$s$ unit selling price per item;

$c$ unit purchasing price per item;

$h_{m}$ unit holding cost per item for raw materials in raw materials warehouse;

$h_{o}$ unit holding cost per item for product in the OW;

$h_{r}$ unit holding cost per item for product in the RW;

$I_{p}$ interest rate payable per \$ unit time (year);

$I_{e}$ interest rate earned per $\$$ unit time (year); 
$t_{s}$ time in years at which production stops;

$M$ the manufacturer's trade credit offered by the supplier;

$N$ the customer's trade credit period offered by the manufacturer;

$W$ storage capacity of the $\mathrm{OW}$;

$t w_{i}$ time when the inventory level increases to $W$ during the production period;

$t w_{d}$ time when the inventory level decreases to $W$ during the production cease period is

$$
T-\frac{W}{D}
$$

$t w_{d}-t w_{i}$ time of rented warehouse is

$$
\left\{\begin{array}{ll}
\frac{D T \rho-W}{P-D}+\frac{D T \rho-W}{D}, & \text { if } D T \rho>W \\
0, & \text { if } D T \rho \leq W
\end{array} ;\right.
$$

$\operatorname{TRC}(T)$ total relevant cost per unit ti when $T>0$;

$T^{*}$ optimal solution of $\operatorname{TRC}(T)$.

\subsection{Assumptions}

1) Demand rate $D$ is known and constant. me of the model.

2) Production rate $P$ is known and constant, $P>D$.

3) Shortages are not allowed.

4) A single item is considered.

5) Time period is infinite.

6) $h_{r} \geq h_{o} \geq h_{m}, M \geq N$ and $s \geq c$.

7) Storage capacity of raw materials warehouse is unlimited.

8) If the order quantity is larger than manufacturer s OW storage capacity, then the manufacturer will rent an RW in which storage capacity is unlimited. When demand occurs, it first is replenished from the RW which storages those exceeding items. RW takes first in last out (FILO).

9) During the period the account is not settled, generated sales revenue is deposited in and interest-bearing account.

a) When $M \leq T$, the account is settled at $T=M$, the manufacturer pays off all units sold, keeps his or her profits, and starts paying for the higher interest payable on the items in stock with rate $I_{p}$.

b) When $T \leq M$, the account is settled at $T=M$ and the manufacturer does not need to pay any interest payable.

10) If a customer buys an item from the manufacturer at time $t \in[0, T]$, then the customer will have a trade credit period $N-t$ and make the payment at time $N$.

11) The manufacturer can accumulate revenue and earn interest after his or her customer pays the amount of the purchasing cost to the manufacturer until the end of the trade credit period offered by the supplier. In other words, the manufacturer can accumulate revenue and earn interest during the period from 
$N$ to $M$ with rate $I_{e}$ under the condition of trade credit.

12) The manufacturer keeps the profit for the use in the other activities.

\subsection{Model}

The model considers three stages of a supply chain system. This paper supposes that the supplier prepares raw materials for production, and the quantity of raw materials is expected to decrease with time (from time 0 to $t_{s}$ ). The quantity of products is expected to increase with time up to the maximum inventory level (from 0 to $t_{s}$ ); the products are sold on demand at the same time. After production stops (at time $t_{s}$ ), the products are sold only on demand until the quantity reaches zero (at time $T$ ), as shown in Figure 1.

\section{The Annual Total Relevant Cost}

The annual total relevant cost consists of the following element.

As shown in Figure 1, the raw material inventory level can be described by the following formulas, and we set the time in years at which production stops $t_{s}$, the optimal order size $Q$ and storage maximum $L_{\max }$ :

$$
\begin{gathered}
(P-D) t_{s}-D\left(T-t_{s}\right)=0, \\
t_{s}=\frac{D T}{P} . \\
\frac{0-Q}{t_{s}-0}=-P, 0 \leq t \leq T .
\end{gathered}
$$

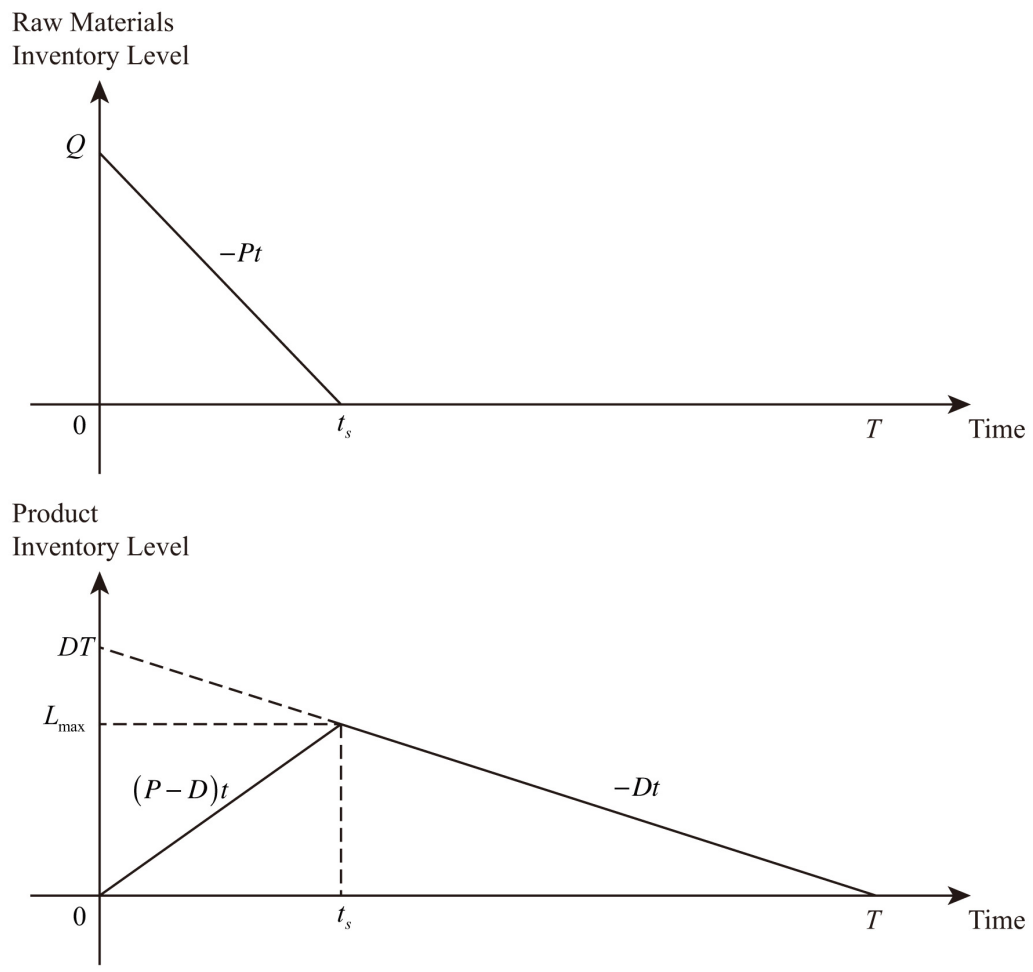

Figure 1. Raw materials and product inventory level. 


$$
\begin{gathered}
Q=P t_{s}=D T . \\
L_{\max }=(P-D) \times t_{s}=(P-D) \frac{D T}{P}=D T \rho .
\end{gathered}
$$

\subsection{Annual Ordering Cost}

Annual ordering cost is

$$
\frac{A}{T}
$$

\subsection{Annual Purchasing Cost}

Annual purchasing cost is

$$
c Q \times \frac{1}{T}=c D .
$$

\subsection{Annual Holding Cost}

Annual holding cost

1) As shown in Figure 1, annual holding cost of raw materials is

$$
h_{m} \times \frac{Q \times t_{s}}{2} \times \frac{1}{T}=h_{m} \times \frac{D T \times \frac{D T}{P}}{2} \times \frac{1}{T}=\frac{D^{2} T h_{m}}{2 P} .
$$

2) Two cases occur in annual holding costs of owned warehouse.

a) $D T \rho \leq W$, as shown in Figure 2 .

Annual holding cost in owned warehouse is

$$
h_{o} \times \frac{T \times L_{\max }}{2} \times \frac{1}{T}=\frac{D T h_{o} \rho}{2} .
$$

b) $D T \rho>W$, as shown in Figure 3 .

Annual holding cost in owned warehouse is

$$
h_{o} \times \frac{\left[\left(t w_{d}-t w_{i}\right)+T\right] W}{2} \times \frac{1}{T}=W h_{o}-\frac{W^{2} h_{o}}{2 D T \rho} .
$$

3) Two cases occur in annual holding costs of rented warehouse.

a) $D T \rho \leq W$, as shown in Figure 2 .

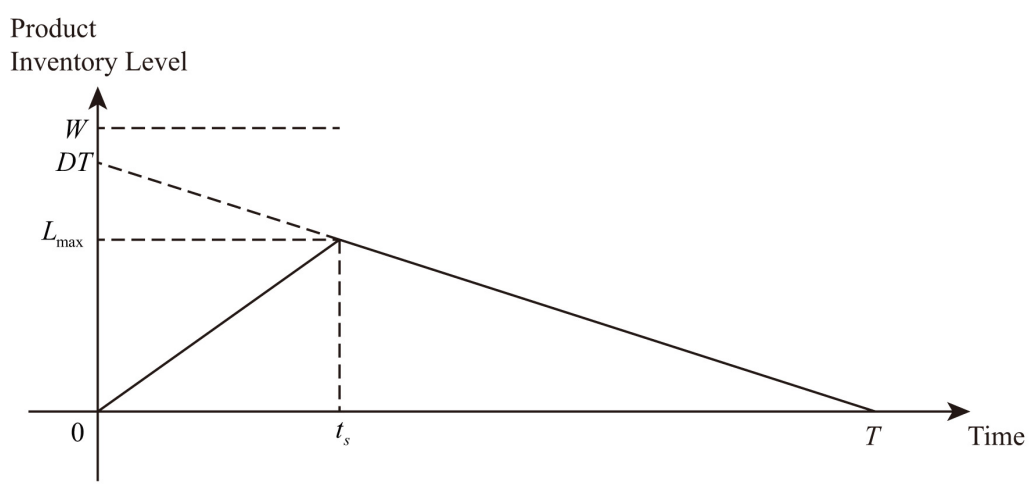

Figure 2. Annual holding cost when $D T \rho \leq W$. 


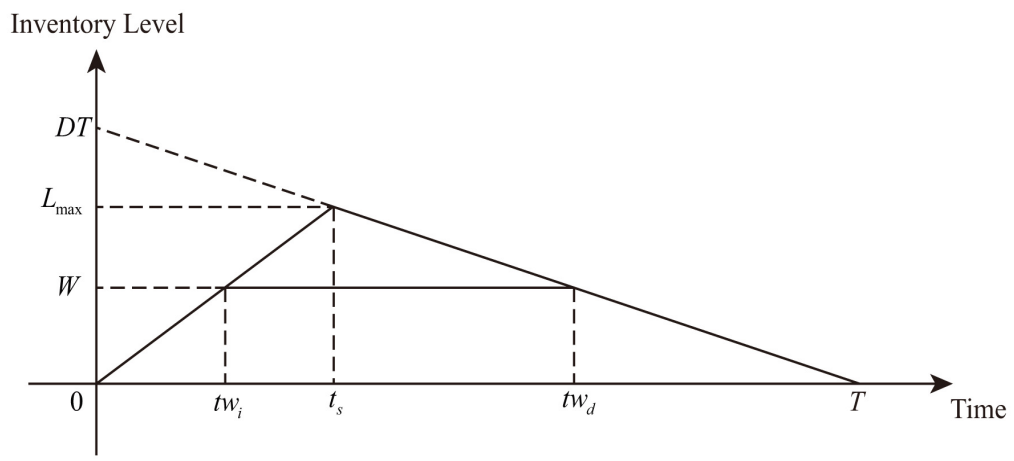

Figure 3. Annual holding cost when $D T \rho>W$.

Annual holding cost in rented warehouse is

0.

b) DT $\rho>W$, as shown in Figure 3 .

Annual holding cost in rented warehouse is

$$
h_{r} \times \frac{\left(t w_{d}-t w_{i}\right) \times\left(L_{\max }-W\right)}{2} \times \frac{1}{T}=\frac{h_{r}(D T \rho-W)^{2}}{2 D T \rho} .
$$

\subsection{Annual Interest Payable}

Four cases to occur in costs of annual interest payable for the items kept in stock.

1) $0<T \leq N$.

Annual interest payable is

0 .

2) $N \leq T \leq M$.

Annual interest payable is

0.

3) $M \leq T \leq \frac{P M}{D}$, as shown in Figure 4 .

Annual interest payable is

$$
C I_{p} \times\left(\frac{(T-M) \times D(T-M)}{2}\right) \times \frac{1}{T}=\frac{c I_{p} D(T-M)^{2}}{2 T} .
$$

4) $M \leq \frac{P M}{D} \leq T$, as shown in Figure 5 .

Annual interest payable is

$$
C I_{p} \times\left(\frac{T \times D T \rho}{2}-\frac{M \times(P-D) M}{2}\right) \times \frac{1}{T}=\frac{C I_{p} \rho\left(D T^{2}-P M^{2}\right)}{2 T} .
$$

\subsection{Annual Interest Earned}

Three cases to occur in annual interest earned.

1) $0<T \leq N$, as shown in Figure 6 . 


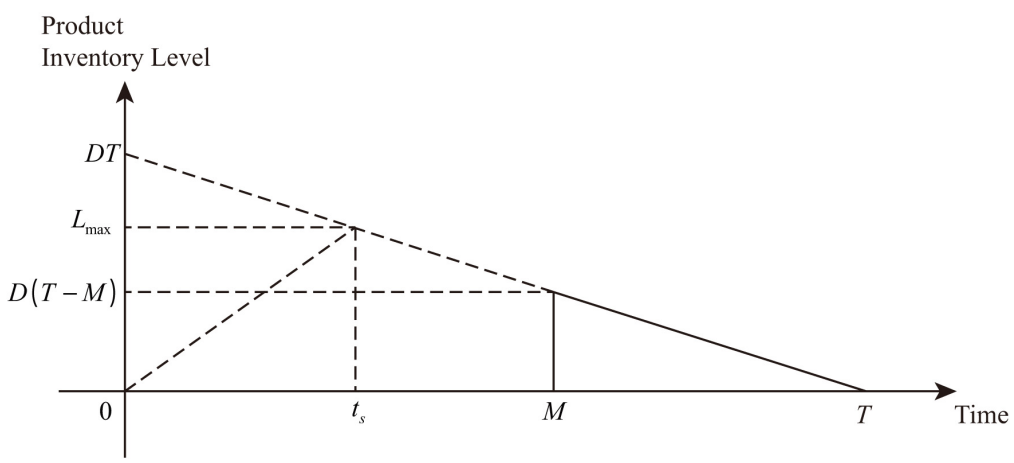

Figure 4. Annual interest payable when $M \leq T \leq \frac{P M}{D}$.

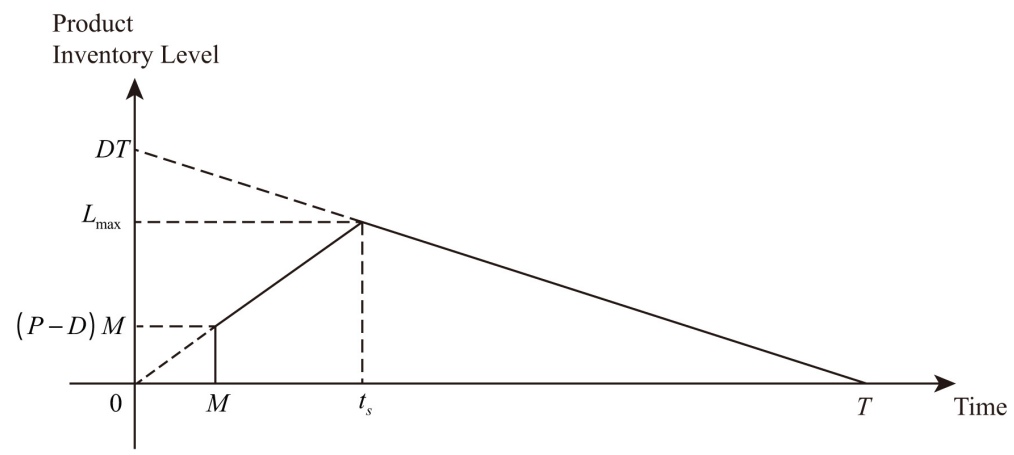

Figure 5. Annual interest payable when $M \leq \frac{P M}{D} \leq T$.

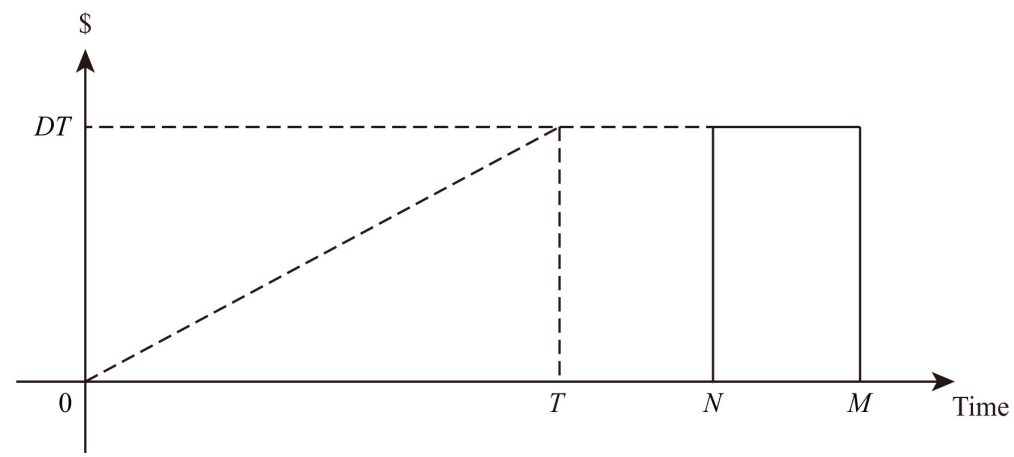

Figure 6. Annual interest earned when $0<T \leq N$.

Annual interest earned is

$$
s I_{e} \times D T(M-N) \times \frac{1}{T}=s I_{e} D(M-N) .
$$

2) $N \leq T \leq M$, as shown in Figure 7 .

Annual interest earned is

$$
\begin{aligned}
& s I_{e} \times\left(\frac{(D N+D T)(T-N)}{2}+D T(M-T)\right) \times \frac{1}{T} \\
& =\frac{s I_{e} D\left(2 M T-N^{2}-T^{2}\right)}{2 T} .
\end{aligned}
$$




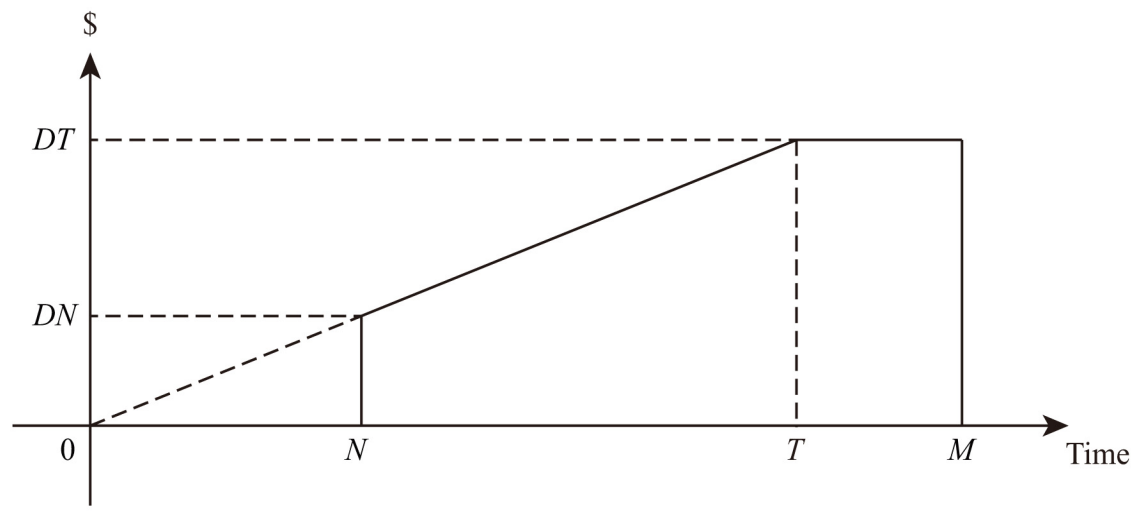

Figure 7. Annual interest earned when $N \leq T \leq M$.

3) $N \leq M \leq T$, as shown in Figure 8 .

Annual interest earned is

$$
s I_{e} \times\left(\frac{(D N+D M)(M-N)}{2}\right) \times \frac{1}{T}=\frac{s I_{e} D\left(M^{2}-N^{2}\right)}{2 T} .
$$

\subsection{The Annual Total Relevant Cost}

From the above arguments, the annual total relevant cost for the manufacturer can be expressed as $\operatorname{TRC}(T)$ = annual ordering cost + annual purchasing cost + annual holding cost + annual interest payable + annual interest earned.

Because storage capacity $W=D T \rho$, there are four cases arise:

1) $\frac{W}{D \rho}<N$,

2) $N \leq \frac{W}{D \rho}<M$,

3) $M \leq \frac{W}{D \rho}<\frac{P M}{D}$,

4) $\frac{P M}{D} \leq \frac{W}{D \rho}$.

Case 1. $\frac{W}{D \rho}<N$.

According to Equations (1)-(17), the annual total relevant cost $T R C(T)$ can be expressed by

$$
T R C(T)= \begin{cases}T R C_{1}(T), & \text { if } 0<T<\frac{W}{D \rho} \\ T R C_{2}(T), & \text { if } \frac{W}{D \rho} \leq T<N \\ T R C_{3}(T), & \text { if } N \leq T<M \\ T R C_{4}(T), & \text { if } M \leq T<\frac{P M}{D} \\ T R C_{5}(T), & \text { if } \frac{P M}{D} \leq T\end{cases}
$$




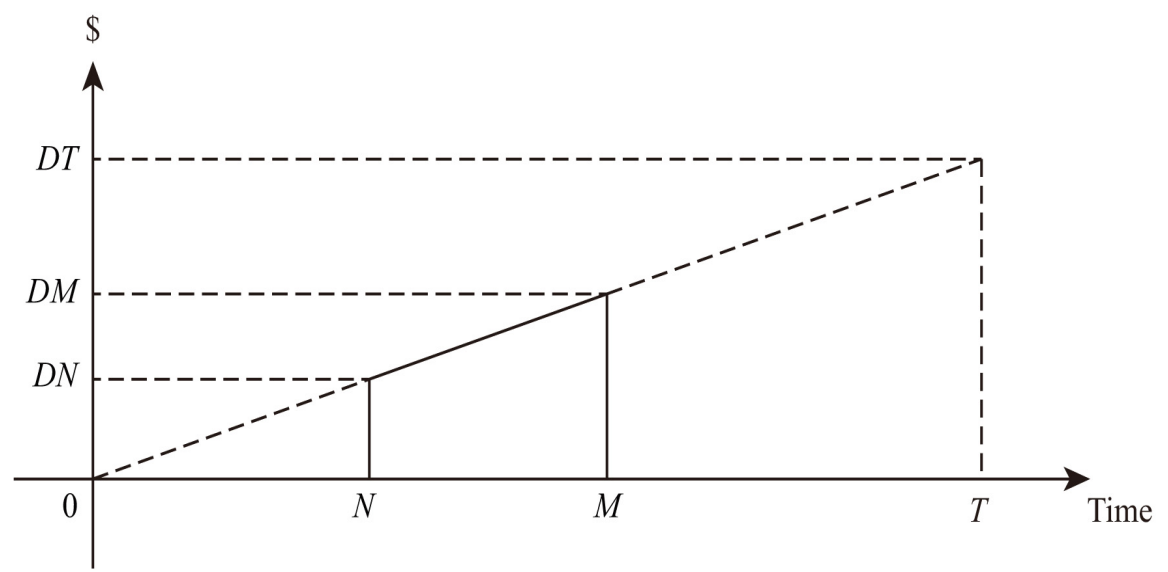

Figure 8. Annual interest earned when $N \leq M \leq T$.

where

$$
\begin{aligned}
T R C_{1}(T) & =\frac{A}{T}+c D+\frac{D^{2} T h_{m}}{2 P}+\frac{D T h_{o} \rho}{2}-s I_{e} D(M-N), \\
T R C_{2}(T)= & \frac{A}{T}+c D+\frac{D^{2} T h_{m}}{2 P}+W h_{o}-\frac{W^{2} h_{o}}{2 D T \rho}+\frac{h_{r}(D T \rho-W)^{2}}{2 D T \rho} \\
& -s I_{e} D(M-N), \\
T R C_{3}(T)= & \frac{A}{T}+c D+\frac{D^{2} T h_{m}}{2 P}+W h_{o}-\frac{W^{2} h_{o}}{2 D T \rho}+\frac{h_{r}(D T \rho-W)^{2}}{2 D T \rho} \\
& -\frac{s I_{e} D\left(2 M T-N^{2}-T^{2}\right)}{2 T}, \\
T R C_{4}(T)= & \frac{A}{T}+c D+\frac{D^{2} T h_{m}}{2 P}+W h_{o}-\frac{W^{2} h_{o}}{2 D T \rho}+\frac{h_{r}(D T \rho-W)^{2}}{2 D T \rho} \\
& +\frac{c I_{p} D(T-M)^{2}}{2 T}-\frac{s I_{e} D\left(M^{2}-N^{2}\right)}{2 T}, \\
& +\frac{c I_{p} \rho\left(D T^{2}-P M^{2}\right)}{2 T}-\frac{s I_{e} D\left(M^{2}-N^{2}\right)}{2 T} . \\
T R C_{5}(T)= & \frac{A}{T}+c D+\frac{D^{2} T h_{m}}{2 P}+W h_{o}-\frac{W_{o}}{2 D T \rho}+\frac{h_{r}(D T \rho-W)^{2}}{2 D T \rho} \\
&
\end{aligned}
$$

$\operatorname{TRC}(T)$ is continuous at $T, T \in[0, \infty)$ because of $T R C_{1}\left(\frac{W}{D \rho}\right)=T R C_{2}\left(\frac{W}{D \rho}\right), T R C_{2}(N)=T R C_{3}(N), T R C_{3}(M)=T R C_{4}(M)$, $\operatorname{TRC}_{4}\left(\frac{P M}{D}\right)=T R C_{5}\left(\frac{P M}{D}\right)$.

Case 2. $N \leq \frac{W}{D \rho}<M$.

According to Equations (1)-(17), the annual total relevant cost $T R C(T)$ can be expressed by 


$$
\operatorname{TRC}(T)= \begin{cases}T R C_{1}(T), & \text { if } 0<T<N \\ T R C_{6}(T), & \text { if } N \leq T<\frac{W}{D \rho} \\ T R C_{3}(T), & \text { if } \frac{W}{D \rho} \leq T<M \\ T R C_{4}(T), & \text { if } M \leq T<\frac{P M}{D} \\ T R C_{5}(T), & \text { if } \frac{P M}{D} \leq T\end{cases}
$$

where

$$
T R C_{6}(T)=\frac{A}{T}+c D+\frac{D^{2} T h_{m}}{2 P}+\frac{D T h_{o} \rho}{2}-\frac{s I_{e} D\left(2 M T-N^{2}-T^{2}\right)}{2 T} .
$$

$\operatorname{TRC}(T)$ is continuous at $T, T \in[0, \infty)$ because of $T R C_{1}(N)=T R C_{6}(N)$, $\operatorname{TRC}_{6}\left(\frac{W}{D \rho}\right)=T R C_{3}\left(\frac{W}{D \rho}\right), T R C_{3}(M)=T R C_{4}(M)$, $\operatorname{TRC}_{4}\left(\frac{P M}{D}\right)=T R C_{5}\left(\frac{P M}{D}\right)$.

Case 3. $M \leq \frac{W}{D \rho}<\frac{P M}{D}$.

According to Equations (1)-(17), the annual total relevant cost $T R C(T)$ can be expressed by

$$
\operatorname{TRC}(T)= \begin{cases}T R C_{1}(T), & \text { if } 0<T<N \\ T R C_{6}(T), & \text { if } N \leq T<M \\ T R C_{7}(T), & \text { if } M \leq T<\frac{W}{D \rho} \\ T R C_{4}(T), & \text { if } \frac{W}{D \rho} \leq T<\frac{P M}{D} \\ T R C_{5}(T), & \text { if } \frac{P M}{D} \leq T\end{cases}
$$

where

$$
\begin{aligned}
T R C_{7}(T)= & \frac{A}{T}+c D+\frac{D^{2} T h_{m}}{2 P}+\frac{D T h_{o} \rho}{2}+\frac{c I_{p} D(T-M)^{2}}{2 T} \\
& -\frac{s I_{e} D\left(M^{2}-N^{2}\right)}{2 T} .
\end{aligned}
$$

$\operatorname{TRC}(T)$ is continuous at $T, T \in[0, \infty)$ because of $\operatorname{TRC}_{1}(N)=T R C_{6}(N)$, $T R C_{6}(M)=T R C_{7}(M), T R C_{7}\left(\frac{W}{D \rho}\right)=T R C_{4}\left(\frac{W}{D \rho}\right)$, $\operatorname{TRC}_{4}\left(\frac{P M}{D}\right)=T R C_{5}\left(\frac{P M}{D}\right)$.

Case 4. $\frac{P M}{D} \leq \frac{W}{D \rho}$. 
According to Equations (1)-(17), the annual total relevant cost $T R C(T)$ can be expressed by

$$
\operatorname{TRC}(T)= \begin{cases}T R C_{1}(T), & \text { if } 0<T<N \\ T R C_{6}(T), & \text { if } N \leq T<M \\ T R C_{7}(T), & \text { if } M \leq T<\frac{P M}{D} \\ T R C_{8}(T), & \text { if } \frac{P M}{D} \leq T<\frac{W}{D \rho} \\ T R C_{5}(T), & \text { if } \frac{W}{D \rho} \leq T\end{cases}
$$

where

$$
\begin{aligned}
T R C_{8}(T)= & \frac{A}{T}+c D+\frac{D^{2} T h_{m}}{2 P}+\frac{D T h_{o} \rho}{2}+\frac{c I_{p} \rho\left(D T^{2}-P M^{2}\right)}{2 T} \\
& -\frac{s I_{e} D\left(M^{2}-N^{2}\right)}{2 T} .
\end{aligned}
$$

$\operatorname{TRC}(T)$ is continuous at $T, T \in[0, \infty)$ because of $T R C_{1}(N)=T R C_{6}(N)$, $T R C_{6}(M)=T R C_{7}(M), T R C_{7}\left(\frac{P M}{D}\right)=T R C_{8}\left(\frac{P M}{D}\right)$, $\operatorname{TRC}_{8}\left(\frac{W}{D \rho}\right)=\operatorname{TR} C_{5}\left(\frac{W}{D \rho}\right)$.

For convenience, all $T R C_{i}(T)(i=1 \sim 8)$ are defined on $T>0$.

\section{The Convexity of $\operatorname{TRC}_{i}(T)(i=1 \sim 8)$}

Equations (19)-(23), (25), (27) and (29) yield the first order and second-order derivatives as follows.

$$
\begin{gathered}
T R C_{1}^{\prime}(T)=\frac{-A}{T^{2}}+\frac{D}{2}\left(\frac{D h_{m}}{P}+\rho h_{o}\right), \\
T R C_{1}^{\prime \prime}(T)=\frac{2 A}{T^{3}}>0, \\
T R C_{2}^{\prime}(T)=\frac{-2 A+\frac{W^{2}\left(h_{o}-h_{r}\right)}{D \rho}}{2 T^{2}}+\frac{D}{2}\left(\frac{D h_{m}}{P}+\rho h_{r}\right), \\
T R C_{3}^{\prime}(T)=\frac{-2 A+\frac{W^{2}\left(h_{o}-h_{r}\right)}{D \rho}-s I_{e} D N^{2}}{\left.2 T^{2}-h_{o}\right)}-\frac{D}{D \rho}\left(\frac{D h_{m}}{P}+\rho h_{r}+s I_{e}\right), \\
T R C_{2}^{\prime \prime}(T)=\frac{T^{3}}{2 A+\frac{W^{2}\left(h_{r}-h_{o}\right)}{D \rho}+s I_{e} D N^{2}}>0, \\
T^{3}
\end{gathered}
$$




$$
\begin{aligned}
& T R C_{4}^{\prime}(T)=\frac{-2 A+\frac{W^{2}\left(h_{o}-h_{r}\right)}{D \rho}+D M^{2}\left(s I_{e}-c I_{p}\right)-s I_{e} D N^{2}}{2 T^{2}} \\
& +\frac{D}{2}\left(\frac{D h_{m}}{P}+\rho h_{r}+c I_{p}\right) \\
& T R C_{4}^{\prime \prime}(T)=\frac{2 A+\frac{W^{2}\left(h_{r}-h_{o}\right)}{D \rho}+D M^{2}\left(c I_{p}-s I_{e}\right)+s I_{e} D N^{2}}{T^{3}}, \\
& T R C_{5}^{\prime}(T)=\frac{-2 A+\frac{W^{2}\left(h_{o}-h_{r}\right)}{D \rho}+D M^{2}\left(s I_{e}-c I_{p}\right)-s I_{e} D N^{2}+c I_{p} P M^{2}}{2 T^{2}} \\
& +\frac{D}{2}\left(\frac{D h_{m}}{P}+\rho\left(h_{r}+c I_{p}\right)\right) \\
& T R C_{5}^{\prime \prime}(T)=\frac{2 A+\frac{W^{2}\left(h_{r}-h_{o}\right)}{D \rho}+D M^{2}\left(c I_{p}-s I_{e}\right)+s I_{e} D N^{2}-c I_{p} P M^{2}}{T^{3}}, \\
& T R C_{6}^{\prime}(T)=\frac{-2 A-s I_{e} D N^{2}}{2 T^{2}}+\frac{D}{2}\left(\frac{D h_{m}}{P}+\rho h_{o}+s I_{e}\right), \\
& T R C_{6}^{\prime \prime}(T)=\frac{2 A+s I_{e} D N^{2}}{T^{3}}>0, \\
& T R C_{7}^{\prime}(T)=\frac{-2 A+D M^{2}\left(s I_{e}-c I_{p}\right)-s I_{e} D N^{2}}{2 T^{2}}+\frac{D}{2}\left(\frac{D h_{m}}{P}+\rho h_{o}+c I_{p}\right), \\
& T R C_{7}^{\prime \prime}(T)=\frac{2 A+D M^{2}\left(c I_{p}-s I_{e}\right)+s I_{e} D N^{2}}{T^{3}}, \\
& T R C_{8}^{\prime}(T)=\frac{-2 A+D M^{2}\left(s I_{e}-c I_{p}\right)-s I_{e} D N^{2}+c I_{p} P M^{2}}{2 T^{2}} \\
& +\frac{D}{2}\left(\frac{D h_{m}}{P}+\rho\left(h_{o}+c I_{p}\right)\right),
\end{aligned}
$$

and

$$
T R C_{8}^{\prime \prime}(T)=\frac{2 A+D M^{2}\left(c I_{p}-s I_{e}\right)+s I_{e} D N^{2}-c I_{p} P M^{2}}{T^{3}} .
$$

Let

$$
\begin{gathered}
G_{4}=2 A+\frac{W^{2}\left(h_{r}-h_{o}\right)}{D \rho}+D M^{2}\left(c I_{p}-s I_{e}\right)+s I_{e} D N^{2}, \\
G_{5}=2 A+\frac{W^{2}\left(h_{r}-h_{o}\right)}{D \rho}+D M^{2}\left(c I_{p}-s I_{e}\right)+s I_{e} D N^{2}-c I_{p} P M^{2}, \\
G_{7}=2 A+D M^{2}\left(c I_{p}-s I_{e}\right)+s I_{e} D N^{2},
\end{gathered}
$$

and

$$
G_{8}=2 A+D M^{2}\left(c I_{p}-s I_{e}\right)+s I_{e} D N^{2}-c I_{p} P M^{2} .
$$


Equations (46)-(49) imply

$$
G_{8}<G_{5}<G_{4} \text {, }
$$

and

$$
G_{8}<G_{7}<G_{4} .
$$

Equations (30)-(45) reveal the following results.

Lemma 1. 1) $T R C_{i}(T)$ is convex on $T>0$ if $i=1,2,3,6$.

2) $T_{R} C_{i}(T)$ is convex on $T>0$ if $G_{i}>0$. Otherwise $T R C_{i}^{\prime}(T)$ is increasing on $T>0$ for all $i=4,5,7,8$. Solving

$$
T R C_{i}^{\prime}(T)=0, i=1 \sim 8
$$

then

$$
\begin{aligned}
& T_{1}^{*}=\sqrt{\frac{2 A}{D\left(\frac{D h_{m}}{P}+\rho h_{o}\right)}}, \\
& T_{2}^{*}=\sqrt{\frac{2 A+\frac{W^{2}\left(h_{r}-h_{o}\right)}{D \rho}}{D\left(\frac{D h_{m}}{P}+\rho h_{r}\right)}}, \\
& T_{3}^{*}=\sqrt{\frac{2 A+\frac{W^{2}\left(h_{r}-h_{o}\right)}{D \rho}+s I_{e} D N^{2}}{D\left(\frac{D h_{m}}{P}+\rho h_{r}+s I_{e}\right)}}, \\
& T_{4}^{*}=\sqrt{\frac{2 A+\frac{W^{2}\left(h_{r}-h_{o}\right)}{D \rho}+D M^{2}\left(c I_{p}-s I_{e}\right)+s I_{e} D N^{2}}{D\left(\frac{D h_{m}}{P}+\rho h_{r}+c I_{p}\right)}} \text { if } G_{4}>0, \\
& T_{5}^{*}=\sqrt{\frac{2 A+\frac{W^{2}\left(h_{r}-h_{o}\right)}{D \rho}+D M^{2}\left(c I_{p}-s I_{e}\right)+s I_{e} D N^{2}-c I_{p} M^{2}}{D\left(\frac{D h_{m}}{P}+\rho\left(h_{r}+c I_{p}\right)\right)}} \text { if } G_{5}>0, \\
& T_{6}^{*}=\sqrt{\frac{2 A+s I_{e} D N^{2}}{D\left(\frac{D h_{m}}{P}+\rho h_{o}+s I_{e}\right)}}, \\
& T_{7}^{*}=\sqrt{\frac{2 A+D M^{2}\left(c I_{p}-s I_{e}\right)+s I_{e} D N^{2}}{D\left(\frac{D h_{m}}{P}+\rho h_{o}+c I_{p}\right)}} \text { if } G_{7}>0 \text {, }
\end{aligned}
$$

and

$$
T_{8}^{*}=\sqrt{\frac{2 A+D M^{2}\left(c I_{p}-s I_{e}\right)+s I_{e} D N^{2}-c I_{p} M^{2}}{D\left(\frac{D h_{m}}{P}+\rho\left(h_{o}+c I_{p}\right)\right)}} \text { if } G_{8}>0,
$$


are the respective solutions of Equation (52). Furthermore, if $T_{i}^{*}$ exists, then $T R C_{i}(T)$ is convex on $T>0$ and

$$
T R C_{i}^{\prime}(T)= \begin{cases}<0 & \text { if } 0<T<T_{i}^{*} \\ =0 & \text { if } T=T_{i}^{*} \\ >0 & \text { if } T_{i}^{*}<T\end{cases}
$$

Equations (61a)-(61c) imply that $T R C_{i}(T)$ is decreasing on $\left(0, T_{i}^{*}\right]$ and increasing on $\left[T_{i}^{*}, \infty\right)$ for all $i=1 \sim 8$.

\section{Numbers $\Delta_{i j}$}

Case 1. $\frac{W}{D \rho}<N$.

Equations (30), (32), (34), (36) and (38) yield

$$
\begin{gathered}
T R C_{1}^{\prime}\left(\frac{W}{D \rho}\right)=T R C_{2}^{\prime}\left(\frac{W}{D \rho}\right)=\frac{\Delta_{12}}{2\left(\frac{W}{D \rho}\right)^{2}}, \\
T R C_{2}^{\prime}(N)=T R C_{3}^{\prime}(N)=\frac{\Delta_{23}}{2 N^{2}}, \\
T R C_{3}^{\prime}(M)=T R C_{4}^{\prime}(M)=\frac{\Delta_{34}}{2 M^{2}}, \\
T R C_{4}^{\prime}\left(\frac{P M}{D}\right)=T R C_{5}^{\prime}\left(\frac{P M}{D}\right)=\frac{\Delta_{45}}{2\left(\frac{P M}{D}\right)^{2}},
\end{gathered}
$$

where

$$
\begin{gathered}
\Delta_{12}=-2 A+D\left(\frac{W}{D \rho}\right)^{2}\left(\frac{D h_{m}}{P}+\rho h_{o}\right), \\
\Delta_{23}=-2 A+\frac{W^{2}\left(h_{o}-h_{r}\right)}{D \rho}+D N^{2}\left(\frac{D h_{m}}{P}+\rho h_{r}\right), \\
\Delta_{34}=-2 A+\frac{W^{2}\left(h_{o}-h_{r}\right)}{D \rho}-s I_{e} D N^{2}+D M^{2}\left(\frac{D h_{m}}{P}+\rho h_{r}+s I_{e}\right), \\
\Delta_{45}=-2 A+\frac{W^{2}\left(h_{o}-h_{r}\right)}{D \rho}+D M^{2}\left(s I_{e}-c I_{p}\right)-s I_{e} D N^{2} \\
+D\left(\frac{P M}{D}\right)^{2}\left(\frac{D h_{m}}{P}+\rho h_{r}+c I_{p}\right)
\end{gathered}
$$

Equations (66)-(69) imply

$$
\Delta_{12}<\Delta_{23}<\Delta_{34}<\Delta_{45} .
$$

Case 2. $N \leq \frac{W}{D \rho}<M$.

Equations (30), (34), (36), (38) and (40) yield 


$$
\begin{gathered}
\operatorname{TRC}_{1}^{\prime}(N)=T R C_{6}^{\prime}(N)=\frac{\Delta_{16}}{2 N^{2}}, \\
T R C_{6}^{\prime}\left(\frac{W}{D \rho}\right)=T R C_{3}^{\prime}\left(\frac{W}{D \rho}\right)=\frac{\Delta_{63}}{2\left(\frac{W}{D \rho}\right)^{2}}, \\
T R C_{3}^{\prime}(M)=T R C_{4}^{\prime}(M)=\frac{\Delta_{34}}{2 M^{2}}, \\
T R C_{4}^{\prime}\left(\frac{P M}{D}\right)=T R C_{5}^{\prime}\left(\frac{P M}{D}\right)=\frac{\Delta_{45}}{2\left(\frac{P M}{D}\right)^{2}},
\end{gathered}
$$

where

$$
\begin{gathered}
\Delta_{16}=-2 A+D N^{2}\left(\frac{D h_{m}}{P}+\rho h_{o}\right), \\
\Delta_{63}=-2 A-s I_{e} D N^{2}+D\left(\frac{W}{D \rho}\right)^{2}\left(\frac{D h_{m}}{P}+\rho h_{o}+s I_{e}\right) .
\end{gathered}
$$

Equations (68), (69), (75) and (76) imply

$$
\Delta_{16} \leq \Delta_{63}<\Delta_{34}<\Delta_{45} .
$$

Case 3. $M \leq \frac{W}{D \rho}<\frac{P M}{D}$.

Equations (30), (36), (38), (40) and (42) yield

$$
\begin{gathered}
T R C_{1}^{\prime}(N)=T R C_{6}^{\prime}(N)=\frac{\Delta_{16}}{2 N^{2},} \\
T R C_{6}^{\prime}(M)=T R C_{7}^{\prime}(M)=\frac{\Delta_{67}}{2 M^{2}}, \\
T R C_{7}^{\prime}\left(\frac{W}{D \rho}\right)=T R C_{4}^{\prime}\left(\frac{W}{D \rho}\right)=\frac{\Delta_{74}}{2\left(\frac{W}{D \rho}\right)^{2}}, \\
T R C_{4}^{\prime}\left(\frac{P M}{D}\right)=T R C_{5}^{\prime}\left(\frac{P M}{D}\right)=\frac{\Delta_{45}}{2\left(\frac{P M}{D}\right)^{2}},
\end{gathered}
$$

where

$$
\begin{gathered}
\Delta_{67}=-2 A-s I_{e} D N^{2}+D M^{2}\left(\frac{D h_{m}}{P}+\rho h_{o}+s I_{e}\right), \\
\Delta_{74}=-2 A+D M^{2}\left(s I_{e}-c I_{p}\right)-s I_{e} D N^{2}+D\left(\frac{W}{D \rho}\right)^{2}\left(\frac{D h_{m}}{P}+\rho h_{o}+c I_{p}\right) .
\end{gathered}
$$

Equations (64), (70), (77) and (78) imply

$$
\Delta_{16} \leq \Delta_{67} \leq \Delta_{74}<\Delta_{45} .
$$

Case 4. $\frac{P M}{D} \leq \frac{W}{D \rho}$. 
Equations (30), (38), (40), (42) and (44) yield

$$
\begin{aligned}
& T R C_{1}^{\prime}(N)=T R C_{6}^{\prime}(N)=\frac{\Delta_{16}}{2 N^{2}}, \\
& T R C_{6}^{\prime}(M)=T R C_{7}^{\prime}(M)=\frac{\Delta_{67}}{2 M^{2}}, \\
& T R C_{7}^{\prime}\left(\frac{P M}{D}\right)=T R C_{8}^{\prime}\left(\frac{P M}{D}\right)=\frac{\Delta_{78}}{2\left(\frac{P M}{D}\right)^{2}}, \\
& T R C_{8}^{\prime}\left(\frac{W}{D \rho}\right)=T R C_{5}^{\prime}\left(\frac{W}{D \rho}\right)=\frac{\Delta_{45}}{2\left(\frac{W}{D \rho}\right)^{2}},
\end{aligned}
$$

where

$$
\begin{aligned}
\Delta_{78}=-2 A+D M^{2}\left(s I_{e}-c I_{p}\right)-s I_{e} D N^{2}+D\left(\frac{P M}{D}\right)^{2}\left(\frac{D h_{m}}{P}+\rho h_{o}+c I_{p}\right), \\
\Delta_{85}=-2 A+D M^{2}\left(s I_{e}-c I_{p}\right)-s I_{e} D N^{2}+c I_{p} P M^{2} \\
+D\left(\frac{W}{D \rho}\right)^{2}\left(\frac{D h_{m}}{P}+\rho\left(h_{o}+c I_{p}\right)\right) .
\end{aligned}
$$

Equations (69), (75), (89) and (90) imply

$$
\Delta_{16} \leq \Delta_{67} \leq \Delta_{78} \leq \Delta_{85} .
$$

Based on the above arguments, the following results holds.

Lemma 2. (A) If $\Delta_{34} \leq 0$, then
(a1) $G_{4}>0$,
(b2) $T_{4}^{*}$ exists,
(c3) $T R C_{4}(T)$ is convex on $T>0$.
(B) If $\Delta_{45} \leq 0$, then
(b1) $G_{4}>0$ and $G_{5}>0$,
(b2) $T_{4}^{*}$ and $T_{5}^{*}$ exist,
(b3) $T R C_{4}(T)$ and $T R C_{5}(T)$ are convex on $T>0$.
(C) If $\Delta_{67} \leq 0$, then
(c1) $G_{7}>0$,
(c2) $T_{7}^{*}$ exists,
(c3) $T R C_{7}(T)$ is convex on $T>0$.
(D) If $\Delta_{74} \leq 0$, then
(d1) $G_{4}>0$ and $G_{7}>0$,
(d2) $T_{4}^{*}$ and $T_{7}^{*}$ exist,
(d3) $T R C_{4}(T)$ and $T R C_{7}(T)$ are convex on $T>0$.
(E) If $\Delta_{78} \leq 0$, then
(e1) $G_{7}>0$ and $G_{8}>0$,
(e2) $T_{7}^{*}$ and $T_{8}^{*}$ exist,
(e3) $T R C_{7}(T)$ and $T R C_{8}(T)$ are convex on $T>0$. 
(F) If $\Delta_{85} \leq 0$, then

(f1) $G_{5}>0$ and $G_{8}>0$,

(f2) $T_{5}^{*}$ and $T_{8}^{*}$ exist,

(f3) $T R C_{5}(T)$ and $T R C_{8}(T)$ are convex on $T>0$.

Proof

(A) (a1) If $\Delta_{34} \leq 0$, then

$$
2 A \geq \frac{W^{2}\left(h_{o}-h_{r}\right)}{D \rho}-s I_{e} D N^{2}+D M^{2}\left(\frac{D h_{m}}{P}+\rho h_{r}+s I_{e}\right) .
$$

Equation (92) implies

$$
G_{4} \geq D M^{2}\left(\frac{D h_{m}}{P}+\rho h_{r}+c I_{p}\right)>0 .
$$

(a2) Equation (56) and lemma 1 imply that $T_{4}^{*}$ exists.

(a3) Equation (37) and lemma 1 imply that $T R C_{4}(T)$ is convex on $T>0$.

(B) (b1) If $\Delta_{45} \leq 0$, then

$$
\begin{aligned}
2 A \geq & \frac{W^{2}\left(h_{o}-h_{r}\right)}{D \rho}+D M^{2}\left(s I_{e}-c I_{p}\right)-s I_{e} D N^{2} \\
& +D\left(\frac{P M}{D}\right)^{2}\left(\frac{D h_{m}}{P}+\rho h_{r}+c I_{p}\right) .
\end{aligned}
$$

Equation (94) implies

$$
G_{5} \geq D\left(\frac{P M}{D}\right)^{2}\left(\frac{D h_{m}}{P}+\rho\left(h_{r}+c I_{p}\right)\right)>0 .
$$

Equations (50) and (95) demonstrate $G_{4}>G_{5}>0$.

(b2) Equations (56), (57) and lemma 1 imply that $T_{4}^{*}$ and $T_{5}^{*}$ exist.

(b3) Equations (37), (39) and lemma 1 imply that $T R C_{4}(T)$ and $T R C_{5}(T)$ are convex on $T>0$.

(C) (c1) If $\Delta_{67} \leq 0$, then

$$
2 A \geq s I_{e} D N^{2}+D M^{2}\left(\frac{D h_{m}}{P}+\rho h_{o}+s I_{e}\right),
$$

Equation (96) implies

$$
G_{7} \geq D M^{2}\left(\frac{D h_{m}}{P}+\rho h_{o}+c I_{p}\right)>0 .
$$

(c2) Equation (59) and lemma 1 imply that $T_{7}^{*}$ exists.

(c3) Equation (43) and lemma 1 imply that $T R C_{7}(T)$ is convex on $T>0$.

(D) (d1) If $\Delta_{74} \leq 0$, then

$$
2 A \geq D M^{2}\left(s I_{e}-c I_{p}\right)-s I_{e} D N^{2}+D\left(\frac{W}{D \rho}\right)^{2}\left(\frac{D h_{m}}{P}+\rho h_{o}+c I_{p}\right) .
$$

Equation (98) implies

$$
G_{7} \geq D\left(\frac{W}{D \rho}\right)^{2}\left(\frac{D h_{m}}{P}+\rho h_{o}+c I_{p}\right)>0 .
$$


Equations (51) and (99) demonstrate $G_{4}>G_{7}>0$.

(d2) Equations (56), (59) and lemma 1 imply that $T_{4}^{*}$ and $T_{7}^{*}$ exist.

(d3) Equations (37), (43) and lemma 1 imply that $T R C_{4}(T)$ and $T R C_{7}(T)$ are convex on $T>0$.

(E) (e1) If $\Delta_{78} \leq 0$, then

$$
2 A \geq D M^{2}\left(s I_{e}-c I_{p}\right)-s I_{e} D N^{2}+D\left(\frac{P M}{D}\right)^{2}\left(\frac{D h_{m}}{P}+\rho h_{o}+c I_{p}\right) .
$$

Equation (100) implies

$$
G_{8} \geq D\left(\frac{P M}{D}\right)^{2}\left(\frac{D h_{m}}{P}+\rho\left(h_{o}+c I_{p}\right)\right)>0 .
$$

Equations (51) and (101) demonstrate $G_{7}>G_{8}>0$.

(e2) Equations (59), (60) and lemma 1 imply that $T_{7}^{*}$ and $T_{8}^{*}$ exist.

(e3) Equations (43), (45) and lemma 1 imply that $T R C_{7}(T)$ and $T R C_{8}(T)$ are convex on $T>0$.

(F) (f1) If $\Delta_{85} \leq 0$, then

$$
\begin{aligned}
2 A \geq & D M^{2}\left(s I_{e}-c I_{p}\right)-s I_{e} D N^{2}+c I_{p} P M^{2} \\
& +D\left(\frac{W}{D \rho}\right)^{2}\left(\frac{D h_{m}}{P}+\rho\left(h_{o}+c I_{p}\right)\right) .
\end{aligned}
$$

Equation (102) implies

$$
G_{8} \geq D\left(\frac{W}{D \rho}\right)^{2}\left(\frac{D h_{m}}{P}+\rho\left(h_{o}+c I_{p}\right)\right)>0 .
$$

Equations (51) and (103) demonstrate $G_{5}>G_{8}>0$.

(f2) Equations (57), (60) and lemma 1 imply that $T_{5}^{*}$ and $T_{8}^{*}$ exist.

(f3) Equations (39), (45) and lemma 1 imply that $T R C_{5}(T)$ and $T R C_{8}(T)$ are convex on $T>0$.

Incorporate the above arguments, we have completed the proof of lemma 2.

\section{The Determination of the Optimal Cycle Time $T^{*}$ of $T R C(T)$}

Theorem 1. Suppose $\frac{W}{D \rho}<N$. Hence,

(A) if $0<\Delta_{12}$, then $\operatorname{TRC}\left(T^{*}\right)=T R C_{1}\left(T_{1}^{*}\right)$ and $T^{*}=T_{1}^{*}$.

(B) if $\Delta_{12} \leq 0<\Delta_{23}$, then $\operatorname{TRC}\left(T^{*}\right)=T R C_{2}\left(T_{2}^{*}\right)$ and $T^{*}=T_{2}^{*}$.

(C) if $\Delta_{23} \leq 0<\Delta_{34}$, then $\operatorname{TRC}\left(T^{*}\right)=T R C_{3}\left(T_{3}^{*}\right)$ and $T^{*}=T_{3}^{*}$.

(D) if $\Delta_{34} \leq 0<\Delta_{45}$, then $\operatorname{TRC}\left(T^{*}\right)=T R C_{4}\left(T_{4}^{*}\right)$ and $T^{*}=T_{4}^{*}$.

(E) if $\Delta_{45} \leq 0$, then $\operatorname{TRC}\left(T^{*}\right)=T R C_{5}\left(T_{5}^{*}\right)$ and $T^{*}=T_{5}^{*}$.

Proof (A) If $0<\Delta_{12}$, then $0<\Delta_{12}<\Delta_{23}<\Delta_{34}<\Delta_{45}$. So, Equations (61a)-(61c), lemma 1 and 2 imply

(a1) $T R C_{1}(T)$ is decreasing on $\left(0, T_{1}^{*}\right]$ and increasing on $\left[T_{1}^{*}, \frac{W}{D \rho}\right]$.

(a2) $T R C_{2}(T)$ is increasing on $\left[\frac{W}{D \rho}, N\right]$. 
(a3) $T R C_{3}(T)$ is increasing on $[N, M]$.

(a4) $T R C_{4}(T)$ is increasing on $\left[M, \frac{P M}{D}\right]$.

(a5) $T R C_{5}(T)$ is increasing on $\left[\frac{P M}{D}, \infty\right)$.

Since $\operatorname{TRC}(T)$ is continuous on $T>0$, Equations (18a)-(18e) and (a1)-(a5) reveal that $\operatorname{TRC}(T)$ is decreasing on $\left(0, T_{1}^{*}\right]$ and increasing on $\left[T_{1}^{*}, \infty\right)$. Hence, $T^{*}=T_{1}^{*}$ and $\operatorname{TRC}\left(T^{*}\right)=T R C_{1}\left(T_{1}^{*}\right)$.

(B) If $\Delta_{12} \leq 0<\Delta_{23}$, then $\Delta_{12} \leq 0<\Delta_{23}<\Delta_{34}<\Delta_{45}$. So, Equations (61a)-(61c), lemma 1 and 2 imply.

(b1) $T R C_{1}(T)$ is decreasing on $\left[0, \frac{W}{D \rho}\right]$.

(b2) $T R C_{2}(T)$ is decreasing on $\left[\frac{W}{D \rho}, T_{2}^{*}\right]$ and increasing on $\left[T_{2}^{*}, N\right]$.

(b3) $T R C_{3}(T)$ is increasing on $[N, M]$.

(b4) $T R C_{4}(T)$ is increasing on $\left[M, \frac{P M}{D}\right]$.

(b5) $T R C_{5}(T)$ is increasing on $\left[\frac{P M}{D}, \infty\right)$.

Since $\operatorname{TRC}(T)$ is continuous on $T>0$, Equations (18a)-(18e) and (a1)-(a5) reveal that $\operatorname{TRC}(T)$ is decreasing on $\left(0, T_{2}^{*}\right]$ and increasing on $\left[T_{2}^{*}, \infty\right)$. Hence, $T^{*}=T_{2}^{*}$ and $\operatorname{TRC}\left(T^{*}\right)=T R C_{2}\left(T_{2}^{*}\right)$.

(C) If $\Delta_{23} \leq 0<\Delta_{34}$, then $\Delta_{12}<\Delta_{23} \leq 0<\Delta_{34}<\Delta_{45}$. So, Equations (61a)-(61c), lemma 1 and 2 imply.

(c1) $T R C_{1}(T)$ is decreasing on $\left[0, \frac{W}{D \rho}\right]$.

(c2) $T_{R C_{2}}(T)$ is decreasing on $\left[\frac{W}{D \rho}, N\right]$.

(c3) $T R C_{3}(T)$ is decreasing on $\left[N, T_{3}^{*}\right]$ and increasing on $\left[T_{3}^{*}, M\right]$.

(c4) $T R C_{4}(T)$ is increasing on $\left[M, \frac{P M}{D}\right]$.

(c5) $T R C_{5}(T)$ is increasing on $\left[\frac{P M}{D}, \infty\right)$.

Since $\operatorname{TRC}(T)$ is continuous on $T>0$, Equations (18a)-(18e) and (c1)-(c5) reveal that $\operatorname{TRC}(T)$ is decreasing on $\left(0, T_{3}^{*}\right]$ and increasing on $\left[T_{3}^{*}, \infty\right)$. Hence, $T^{*}=T_{3}^{*}$ and $\operatorname{TRC}\left(T^{*}\right)=T R C_{3}\left(T_{3}^{*}\right)$.

(D) If $\Delta_{34} \leq 0<\Delta_{45}$, then $\Delta_{12}<\Delta_{23}<\Delta_{34} \leq 0<\Delta_{45}$. So, Equations (61a)-(61c), lemma 1 and 2 imply
(d1) $T R C_{1}(T)$ is decreasing on $\left[0, \frac{W}{D \rho}\right]$.
(d2) $T R C_{2}(T)$ is decreasing on $\left[\frac{W}{D \rho}, N\right]$.
(d3) $T R C_{3}(T)$ is decreasing on $[N, M]$. 
(d4) $T R C_{4}(T)$ is decreasing on $\left[M, T_{4}^{*}\right]$ and increasing on $\left[T_{4}^{*}, \frac{P M}{D}\right]$.

(d5) $T R C_{5}(T)$ is increasing on $\left[\frac{P M}{D}, \infty\right)$.

Since $\operatorname{TRC}(T)$ is continuous on $T>0$, Equations (18a)-(18e) and (d1)-(d5) reveal that $\operatorname{TRC}(T)$ is decreasing on $\left(0, T_{4}^{*}\right]$ and increasing on $\left[T_{4}^{*}, \infty\right)$. Hence, $T^{*}=T_{4}^{*}$ and $\operatorname{TRC}\left(T^{*}\right)=T R C_{4}\left(T_{4}^{*}\right)$.

(E) If $\Delta_{45} \leq 0$, then $\Delta_{12}<\Delta_{23}<\Delta_{34}<\Delta_{45} \leq 0$. So, Equations (61a)-(61c), lemma 1 and 2 imply.

(e1) $T R C_{1}(T)$ is decreasing on $\left[0, \frac{W}{D \rho}\right]$.

(e2) $T R C_{2}(T)$ is decreasing on $\left[\frac{W}{D \rho}, N\right]$.

(e3) $T R C_{3}(T)$ is decreasing on $[N, M]$.

(e4) $T R C_{4}(T)$ is decreasing on $\left[M, T_{4}^{*}\right]$.

(e5) $T R C_{5}(T)$ is decreasing on $\left[\frac{P M}{D}, T_{5}^{*}\right]$ and increasing on $\left[T_{5}^{*}, \infty\right)$.

Since $\operatorname{TRC}(T)$ is continuous on $T>0$, Equations (18a)-(18e) and (e1)-(e5) reveal that $\operatorname{TRC}(T)$ is decreasing on $\left(0, T_{5}^{*}\right]$ and increasing on $\left[T_{5}^{*}, \infty\right)$. Hence, $T^{*}=T_{5}^{*}$ and $\operatorname{TRC}\left(T^{*}\right)=T R C_{5}\left(T_{5}^{*}\right)$.

Incorporating all argument above arguments, we have completed the proof of Theorem 1.

Theorem 2. Suppose $N \leq \frac{W}{D \rho}<M$. Hence,

(A) if $0<\Delta_{16}$, then $\operatorname{TRC}\left(T^{*}\right)=T R C_{1}\left(T_{1}^{*}\right)$ and $T^{*}=T_{1}^{*}$.

(B) if $\Delta_{16} \leq 0<\Delta_{63}$, then $\operatorname{TRC}\left(T^{*}\right)=T R C_{6}\left(T_{6}^{*}\right)$ and $T^{*}=T_{6}^{*}$.

(C) if $\Delta_{63} \leq 0<\Delta_{34}$, then $\operatorname{TRC}\left(T^{*}\right)=T R C_{3}\left(T_{3}^{*}\right)$ and $T^{*}=T_{3}^{*}$.

(D) if $\Delta_{34} \leq 0<\Delta_{45}$, then $\operatorname{TRC}\left(T^{*}\right)=T R C_{4}\left(T_{4}^{*}\right)$ and $T^{*}=T_{4}^{*}$.

(E) if $\Delta_{45} \leq 0$, then $\operatorname{TRC}\left(T^{*}\right)=T R C_{5}\left(T_{5}^{*}\right)$ and $T^{*}=T_{5}^{*}$.

Applying lemmas 1, 2 and Equations (24a)-(24e), the following results hold.

Theorem 3. Suppose $M \leq \frac{W}{D \rho}<\frac{P M}{D}$. Hence,

(A) if $0<\Delta_{16}$, then $\operatorname{TRC}\left(T^{*}\right)=T R C_{1}\left(T_{1}^{*}\right)$ and $T^{*}=T_{1}^{*}$.

(B) if $\Delta_{16} \leq 0<\Delta_{67}$, then $\operatorname{TRC}\left(T^{*}\right)=T R C_{6}\left(T_{6}^{*}\right)$ and $T^{*}=T_{6}^{*}$.

(C) if $\Delta_{67} \leq 0<\Delta_{74}$, then $\operatorname{TRC}\left(T^{*}\right)=T R C_{7}\left(T_{7}^{*}\right)$ and $T^{*}=T_{7}^{*}$.

(D) if $\Delta_{74} \leq 0<\Delta_{45}$, then $\operatorname{TRC}\left(T^{*}\right)=T R C_{4}\left(T_{4}^{*}\right)$ and $T^{*}=T_{4}^{*}$.

(E) if $\Delta_{45} \leq 0$, then $\operatorname{TRC}\left(T^{*}\right)=T R C_{5}\left(T_{5}^{*}\right)$ and $T^{*}=T_{5}^{*}$.

Applying lemmas 1, 2 and Equations (26a)-(26e), the following results hold.

Theorem 4. Suppose $\frac{P M}{D} \leq \frac{W}{D \rho}$. Hence,

(A) if $0<\Delta_{16}$, then $\operatorname{TRC}\left(T^{*}\right)=T R C_{1}\left(T_{1}^{*}\right)$ and $T^{*}=T_{1}^{*}$.

(B) if $\Delta_{16} \leq 0<\Delta_{67}$, then $\operatorname{TRC}\left(T^{*}\right)=T R C_{6}\left(T_{6}^{*}\right)$ and $T^{*}=T_{6}^{*}$.

(C) if $\Delta_{67} \leq 0<\Delta_{78}$, then $\operatorname{TRC}\left(T^{*}\right)=T R C_{7}\left(T_{7}^{*}\right)$ and $T^{*}=T_{7}^{*}$.

(D) if $\Delta_{78} \leq 0<\Delta_{85}$, then $\operatorname{TRC}\left(T^{*}\right)=T R C_{8}\left(T_{8}^{*}\right)$ and $T^{*}=T_{8}^{*}$. 
(E) if $\Delta_{85} \leq 0$, then $\operatorname{TRC}\left(T^{*}\right)=T R C_{5}\left(T_{5}^{*}\right)$ and $T^{*}=T_{5}^{*}$.

Applying lemmas 1, 2 and Equations (28a)-(28e), the following results hold.

\section{Sensitivity Analysis}

We present the sensitivity analysis by Maple 18.00 for determining the unique solution $T_{i}^{*}$ when $T R C_{i}^{\prime}(T)=0, i=1 \sim 8$, given $P=4500$ units/year, $D=3000$ units/year, $A=\$ 1000 /$ order , $s=\$ 30 /$ unit , $c=\$ 10 /$ unit , $h_{m}=\$ 0.5 /$ unit $/$ year,$h_{o}=\$ 1.5 /$ unit $/$ year,$h_{r}=\$ 5 /$ unit $/$ year,$I_{p}=\$ 0.3 /$ year , $I_{e}=\$ 0.08 /$ year,$M=90$ days $=90 / 365$ year,$N=45$ days $=45 / 365$ year , $W=350$ units. We increase/decrease $25 \%$ and $50 \%$ of the parameters simultaneously to execute the sensitivity analysis. Table 1 lists the results of comparing the computational results with those of [9] (Figures 9-12).

\section{Conclusions}

Although traditional models facilitate calculation, they ignore the importance of raw materials. However, the related costs of raw materials directly affect the total relevant costs, thereby generating significant errors in the overall consideration.

Therefore, this paper presents a new inventory model that applies raw materials with two-level of trade credit-the finite replenishment rate and limited storage capacity - by considering the trade credit condition proposed by [7]. Consequently, [9] can be treated as a special case in this paper.

Table 1. Comparison of relative parameters impact to $T^{*}$ and $\operatorname{TRC}\left(T^{*}\right)$ in the sensitivity analysis.

\begin{tabular}{ccccc}
\hline \multirow{2}{*}{ Impact } & \multicolumn{2}{c}{$T$} & \multicolumn{2}{c}{$T R C(T)$} \\
\cline { 2 - 5 } & this paper & {$[9]$} & this paper & {$[9]$} \\
\hline Positive \& Major & $A$ & $A$ & $c$ & $A$ \\
Positive \& Minor & $h_{r}, I_{e}$ & $h_{r}, I_{e}$ & $A, s, h_{m}, h_{o}, I_{p}$ & $s, c, h_{o}, I_{p}$ \\
Negative \& Minor & $S, c, h_{m}, h_{o}$ & $s, c, h_{o}$ & $h_{r}, I_{e}$ & $h_{r}, I_{e}$ \\
Negative \& Major & $I_{p}$ & $I_{p}$ & - & - \\
\hline
\end{tabular}

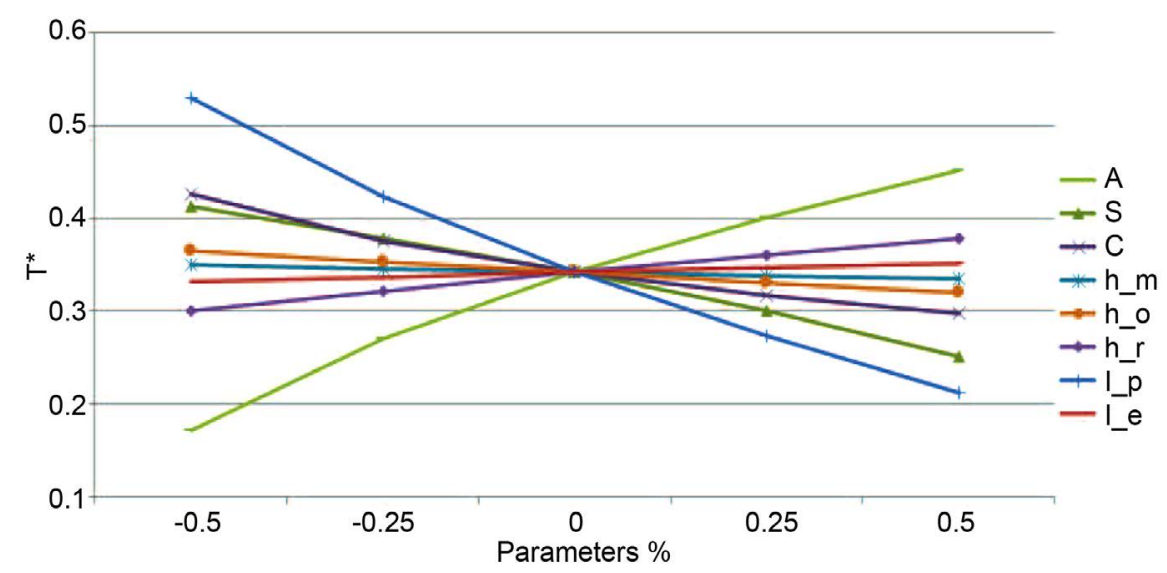

Figure 9. The sensitivity analysis for $T^{*}$ of this paper. 


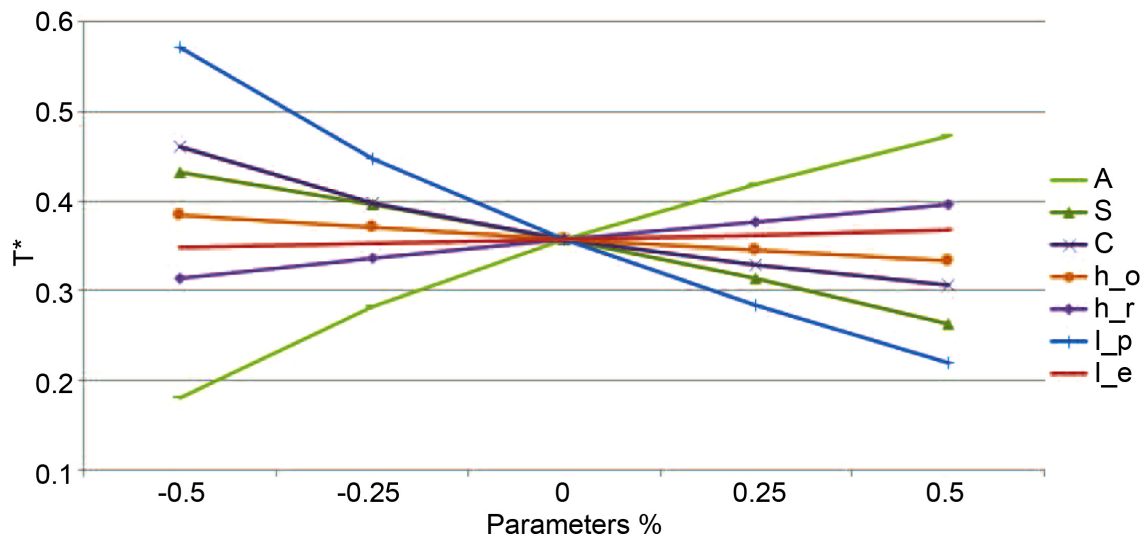

Figure 10. The sensitivity analysis for $T^{*}$ of [9].

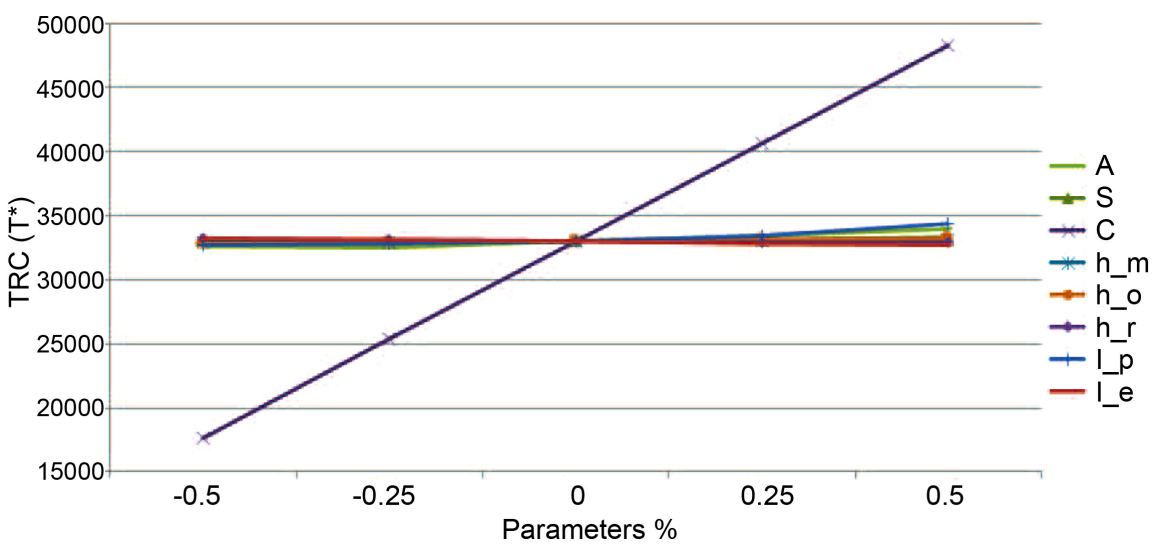

Figure 11. The sensitivity analysis for $\operatorname{TRC}\left(T^{*}\right)$ of this paper.

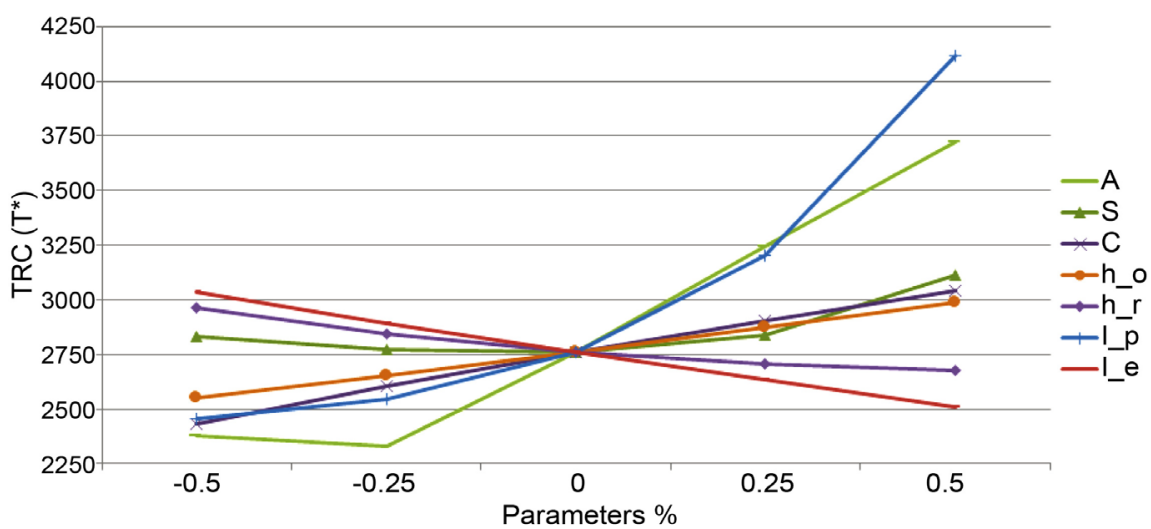

Figure 12. The sensitivity analysis for $\operatorname{TRC}\left(T^{*}\right)$ of [9].

\section{Conflicts of Interest}

The authors declare no conflicts of interest regarding the publication of this paper.

\section{References}

[1] Harris, F.W. (1913) How Many Parts to Make at Once. Factory, the Magazine of 
Management, 10, 135-136,

[2] Taft, E.W. (1918) The Most Economical Production Lot. Iron Age, 101, 1410-1412.

[3] Lin, S.D. (2010) The Optimal Inventory Policy of Production Management. Across-Straits Academic Conference Proceedings in Management Theories and Practices, Jilin, China.

[4] Su, S.M. and Lin, S.D. (2013) The Optimal Inventory Policy of Production Management. Engineering, 5, 9-13. https://doi.org/10.4236/eng.2013.55A002

[5] Su, S.M., Lin, S.D., and Chang, L.F. (2014) The Optimal Inventory Policy for Reusable Items with Random Planning Horizon Considering Present Value. Applied Mathematics, 5, 292-299. https://doi.org/10.4236/am.2014.52030

[6] Goyal, S.K. (1985) Economic Order Quantity under Conditions of Permissible Delay in Payments. Journal of the Operational Research Society, 36, 335-338. https://doi.org/10.1057/jors.1985.56

[7] Huang, Y.F. (2003) Optimal Retailer's Ordering Policies in the EOQ Model under Trade Credit Financing. Journal of the Operational Research Society, 54, 1011-1015. https://doi.org/10.1057/palgrave.jors.2601588

[8] Chung, K.J. (2011) The Simplified Solution Procedures for the Optimal Replenishment Decisions under Two Levels of Trade Credit Policy Depending on the Order Quantity in a Supply Chain System. Expert Systems with Applications, 38, 13482-13486. https://doi.org/10.1016/j.eswa.2011.04.094

[9] Chung, K.J. (2013) The EPQ Model under Conditions of Two Levels of Trade Credit and Limited Storage Capacity in Supply Chain Management. International Journal of Systems Science, 44, 1675-1691. https://doi.org/10.1080/00207721.2012.669864

[10] Kreng, V.B. and Tan, S.J. (2010) The Optimal Replenishment Decisions under Two Levels of Trade Credit Policy Depending on the Order Quantity. Expert Systems with Applications, 37, 5514-5522. https://doi.org/10.1016/j.eswa.2009.12.014

[11] Min, J., Zhou, Y.W. and Zhao, J. (2010) An Inventory Model for Deteriorating Items under Stock-Dependent Demand and Two-Level Trade Credit. Applied Mathematical Modelling, 34, 3273-3285. https://doi.org/10.1016/j.apm.2010.02.019

[12] Yen, G.F., Chung, K.J. and Chen, T.C. (2012) The Optimal Retailer's Ordering Policies with Trade Credit Financing and Limited Storage Capacity in the Supply Chain System. International Journal of Systems Science, 43, 2144-2159. https://doi.org/10.1080/00207721.2011.565133

[13] Srivastava, H.M., Yen, G.F., Lee, A.K., Wu, Y.X. and Lin, S.D. (2018) The Optimal Retailer's EPQ Policies with Two-Level Trade Credit under Alternate Due Date of Payment and Limited Storage Capacity. Applied Mathematics \& Information Sciences, 12, 1073-1089. https://doi.org/10.18576/amis/120602

[14] Hariga, M.A. (2011) Inventory Models for Multi-Warehouse Systems under Fixed and Flexible Space Leasing Contracts. Computers \& Industrial Engineering, 61, 744-751. https://doi.org/10.1016/j.cie.2011.05.006

[15] Hartley, R.V. (1976) Operations Research: A Managerial Emphasis. 1st Edition, Goodyear Publishing, Pacific Palisades, CA.

[16] Liao, J.J., Chung, K.J. and Huang, K.N. (2013) A Deterministic Inventory Model for Deteriorating Items with Two Warehouses and Trade Credit in a Supply Chain System. International Journal of Production Economics, 146, 557-565. https://doi.org/10.1016/j.ijpe.2013.08.001 